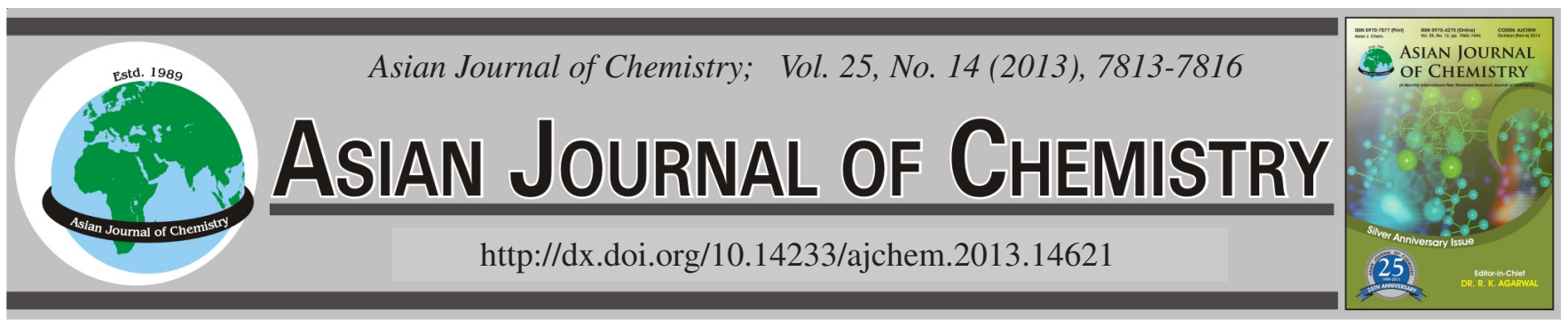

\title{
Molecular Structure and Ordering in Biphenyl Molecules with Strong Polar Group: A Comparative Computational Analysis Between a Mesogen and Non-Mesogens
}

\author{
P. Lakshmi Praveen and Durga P. OJha*
}

School of Physics, Sambalpur University, Jyoti Vihar, -768 019, India

*Corresponding author: E-mail: durga_ojha@ @otmail.com

\begin{abstract}
The systems chosen, 4-heptyl-3'-cyanobiphenyl and 4-heptyl-4'-nitrobiphenyl are non-mesogens having strong polar group. A computational approach of ordering in these non-mesogens has been carried out with respect to translatory and orientational motions. The evaluation of net atomic charges and dipole moments at each atomic center has been carried out using the complete neglect differential overlap (CNDO/ 2) method. The modified Rayleigh-Schrodinger perturbation theory along with the multicentered-multipole expansion method has been employed to evaluate long-range interactions, while a "6-exp" potential function has been assumed for short-range interactions. All possible geometrical arrangements between a molecular pair have been considered and the most favorable stacked and in-plane energy configurations have been obtained. A comparative picture of molecular parameters, such as total energy, binding energy and total dipole moment of the non-mesogens with a mesogen 4-heptyl-4'-cyanobiphenyl has been reported. It is found that, if a suitable functional group is attached/replaced in these systems, so that the length to breadth ratio is altered, the molecule will show a change in the long-range order, the phase transition temperature. This affords a new way of looking at the substitution of suitable functional groups, which may have an effective role on anisotropic molecular interaction and other factors responsible for liquid crystallinity.
\end{abstract}

Key Words: Polar group, CNDO/2 method, Rayleigh-Schrodinger perturbation theory.

\section{INTRODUCTION}

Liquid crystals (LCs) are indeed a new facet in crystal engineering that is intermediary in structure and molecular organization between amorphous liquid state and the solid crystalline state. Liquid crystal research has an interdisciplinary and international pursuit. Recent research on these molecular devices explores new innovations with much attention to the molecular interactions, suitable aromatic groups and functional groups that causes/vanishes liquid crystallinity ${ }^{1,2}$. Such focus can bring a deeper knowledge to ascertain the correlation between structure and properties of liquid crystals. It is necessary not only to study the physicochemical properties but also to have a look at the configuration of substituents, which may have an effective role on anisotropic molecular interaction ${ }^{3}$. The decisive objective of these studies is to determine the macroscopic properties of complex systems, such as liquid crystals from the microscopic quantities. The firm knowledge about the model potential can provide valuable information on physical properties of liquid crystalline materials ${ }^{4,5}$.

The accomplishments of liquid crystal research based on computational studies in advancing the structure and energetic effects of intermolecular interactions are useful in employing molecular models, yield insight into general features of phase equilibria, structure and dynamics of liquid crystals. Many liquid crystal materials have reported based on intermolecular interaction energy calculations using the Rayleigh-Schrodinger perturbation $\operatorname{method}^{6-8}$. These studies were aimed at establishing the anisotropic nature of the pair potential and subsequently finding out the minimum energy configuration of a pair of liquid crystalline molecules. Thus, the main emphasis was laid on finding out the minimum energy with observed crystal structure, the basic motive for ordering of a molecule. It has been observed that the interaction energies for a pair of mesogens indicate the preference of a particular configuration over the other depending on their energy values.

The correlation between structure and mesomorphic properties of molecular systems can be determined by molecular interactions. In general, these interactions are non-central, i.e., they are dependent on the relative orientation/ translation of molecules $^{9,10}$. The role of molecular shape and intermolecular interactions has a sizeable importance in predicting physical properties and structures of liquid crystalline compounds ${ }^{9}$. A significant contribution to the total pair energy can be achieved by the electrostatic interactions in a molecular pair; but more importantly, their anisotropy plays a significant role in 
molecular recognition. The non-bonded interactions involve coulombic and dispersion forces. Many liquid crystal molecules have a permanent dipole moment and, therefore dipoledipole term is also necessary. Higher order multipole terms are thought to influence the bulk structure, but are often excluded due to the lack of the experimental measurements of quadrupolemoments. Molecular interactions can promote or oppose polar organization in mesophases. Thus, adopted model potential has a crucial role in better understanding of mesomorphism.

The systems chosen for present investigation 4-heptyl3'-cyanobiphenyl (7CB3) and 4-heptyl-4'-nitrobiphenyl (7NB4) are not strictly mesogens but seems to be quite interesting since it can be converted into mesogens by attaching/ replacing the suitable substituents. In the present approach, ordering of these non-mesogens has been carried out at an intermediate distance of $8 \AA$ for stacking and $10 \AA$ for inplane interactions. Similarly, a distance of $22 \AA$ has been kept for terminal interactions. The choice of distance has been made to eliminate the possibility of van der Waals contacts completely and to keep the molecules within the short- and mediumrange interactions. The detailed results are reported only for 7CB3. However, the salient features of the other non-mesogen i.e., $7 \mathrm{NB} 4$ are reported with mesogen $7 \mathrm{CB} 4^{10}$.

\section{COMPUTATIONAL APPROACH}

The molecular geometry of 7CB3 and 7NB4 (Fig. 1) has been constructed on the basis of published crystallographic data with the standard values of bond lengths and bond angles ${ }^{11,12}$. In order to achieve the interaction picture of nonmesogens, the following steps have been carried out:

The simplified formula for interaction energy calculations requires the evaluation of atomic net charges and dipole moment components at each atomic centre through an allvalance electron method. In the present computation, the $\mathrm{CNDO} / 2$ method $^{13}$ has been employed to compute the net atomic charge and dipole moment at each atomic centre of the molecule. A detailed computational scheme based on simplified formula provided by Claverie ${ }^{14,15}$ for the evaluation of interaction energy between a molecular pair has been used to calculate the energy for fixed configuration. According to the second order perturbation theory as modified by Caillet and Claverie $^{15,16}$ for intermediate range interactions, the total pair interaction energy of molecules $\left(\mathrm{U}_{\text {pair }}\right)$ is represented as sum of various terms contributing to the total energy:

$$
\mathrm{U}_{\text {pair }}=\mathrm{U}_{\mathrm{el}}+\mathrm{U}_{\text {pol }}+\mathrm{U}_{\text {disp }}+\mathrm{U}_{\text {rep }}
$$

where $\mathrm{U}_{\mathrm{el}}, \mathrm{U}_{\text {pol }}, \mathrm{U}_{\text {disp }}$ and $\mathrm{U}_{\text {rep }}$ are the electrostatic, polarization, dispersion and repulsion energy terms, respectively. Again, electrostatic term is expressed as

$$
\mathrm{U}_{\mathrm{el}}=\mathrm{U}_{\mathrm{QQ}}+\mathrm{U}_{\mathrm{QMI}}+\mathrm{U}_{\mathrm{MIMI}}+\ldots
$$

where $\mathrm{U}_{\mathrm{QQ}}, \mathrm{U}_{\mathrm{QMI}}$ and $\mathrm{U}_{\mathrm{MIMI}}$ etc., are monopole-monopole, monopole-dipole and dipole-dipole terms, respectively. In fact, the inclusion of higher order multipoles does not affect significantly the electrostatic interaction energy and the calculation only up to dipole-dipole term gives satisfactory result $^{16}$. The computation of electrostatic term has, therefore, been restricted only up to dipole-dipole energy term.
In the present computation, the dispersion and short-range repulsion terms are considered together because several semiemperical approach, viz., the Lennard-Jones or Buckingham type approach, actually proceed in this way. Kitaygorodsky introduced a Buckingham formula whose parameters were later modified by Kitaygorodsky and Mirskay ${ }^{17}$ for hydrocarbon molecules and several other molecules and finally gave the expression:

$$
\begin{gathered}
\mathrm{U}_{\mathrm{disp}}+\mathrm{U}_{\text {rep }}=\sum_{\lambda}^{(1)} \sum_{v}^{(2)} \mathrm{U}(\lambda, v) \\
\mathrm{U}(\lambda, v)=\mathrm{K}_{\lambda} \mathrm{K}_{v}\left(-\frac{\mathrm{A}}{\mathrm{Z}^{6}}+\mathrm{Be}^{-\gamma \mathrm{Z}}\right)
\end{gathered}
$$

where $\mathrm{Z}=\mathrm{R}_{\lambda v} / \mathrm{R}^{0}{ }_{\lambda v} ; \mathrm{R}^{0}{ }_{\lambda v}=\left[\left(2 \mathrm{R}^{\mathrm{w}}{ }_{\lambda}\right)\left(2 \mathrm{R}^{\mathrm{w}}{ }_{v}\right)\right]^{1 / 2}$, where $\mathrm{R}^{\mathrm{w}}{ }_{\lambda}$ and $\mathrm{R}^{\mathrm{w}}{ }_{v}$ are the van der Waals radii of atom $\lambda$ and $v$, respectively. The parameters A, B and $\gamma$ do not depend on the atomic species. But $\mathrm{R}_{\lambda v}^{0}$ and factor $\mathrm{K}_{\lambda} \mathrm{K}_{v}$ allows the energy minimum to have different values according to the atomic species involved. The necessary formulae may be found elsewhere ${ }^{18-20}$.

An orthogonal coordinate system is considered to facilitate the above calculation. The origin on an atom has been chosen at almost midpoint of the molecule. The $\mathrm{x}$-axis along a bond parallel to the long molecular axis while the $y$-axis lies in the plane of the molecule and $\mathrm{z}$-axis perpendicular to the molecular plane.

\section{RESULTS AND DISCUSSION}

The molecular geometry of 7CB3 and 7NB4 is shown in Fig. 1. The net charges and dipole moments corresponding to each atomic center are listed in Table-1. A comparative picture of molecular parameters, such as total energy, binding energy and total dipole moment of 7CB3, 7NB4 with a nematogen $7 \mathrm{CB} 4$, is shown in Fig. 2. Atom-based partial charges on heavy atoms of the molecules have been reported in Fig. 3 to analyze the molecular charge distribution, which enables to study the different modes of intermolecular interactions.

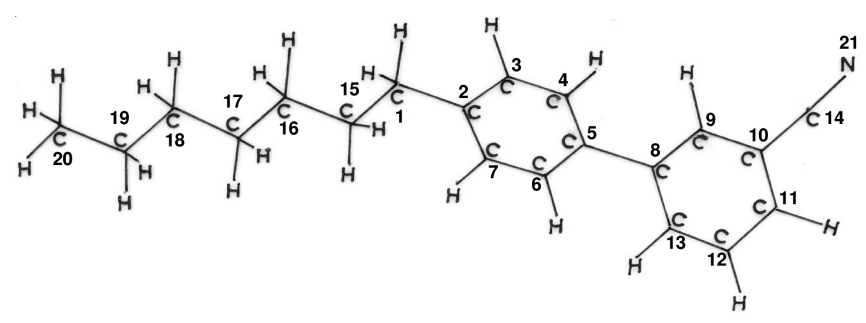

(a)

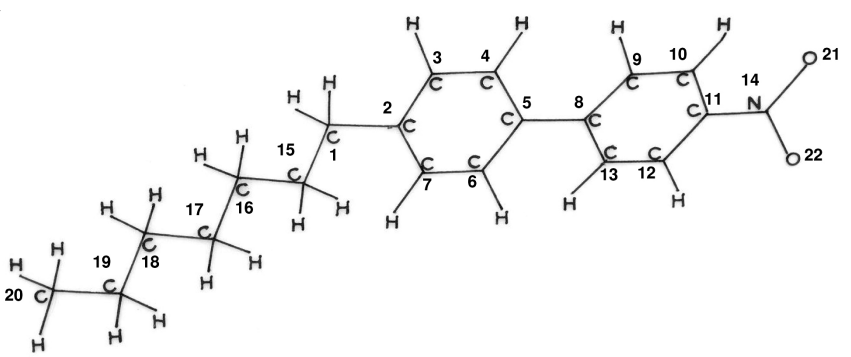

(b)

Fig. 1. Molecular Geometry of (a) 7CB3 and (b) 7NB4 with various atoms 
TABLE-1

MINIMUM ENERGY OBTAINED AFTER REFINEMENT

DURING STACKING AND IN-PLANE INTERACTIONS

BETWEEN A MOLECULAR PAIR OF 7CB3. ENERGY IS EXPRESSED IN kcal/mol

\begin{tabular}{ccc}
\hline Energy terms & Stacking energy & In-plane energy \\
\hline $\mathrm{U}_{\mathrm{QQ}}$ & 0.82 & 0.67 \\
$\mathrm{U}_{\mathrm{QMI}}$ & 0.01 & -0.55 \\
$\mathrm{U}_{\mathrm{MIMI}}$ & -0.52 & -0.41 \\
$\mathrm{U}_{\mathrm{el}}$ & 0.09 & -0.88 \\
$\mathrm{U}_{\text {pol }}$ & -0.87 & -0.96 \\
$\mathrm{U}_{\text {disp }}$ & -19.43 & -8.59 \\
$\mathrm{U}_{\text {rep }}$ & 8.99 & 3.99 \\
$\mathrm{U}_{\text {total }}$ & -13.67 & -7.79 \\
\hline
\end{tabular}

$\mathrm{U}_{\mathrm{QQ}}=$ Monopole-monopole; $\mathrm{U}_{\mathrm{QMI}}=$ monopole-dipole; $\mathrm{U}_{\mathrm{MIMI}}=$ dipoledipole; $\mathrm{U}_{\mathrm{el}}=$ electrostatic; $\mathrm{U}_{\mathrm{pol}}=$ polarization; $\mathrm{U}_{\mathrm{disp}}=$ dispersion; Urep $=$ repulsion and $\mathrm{U}_{\text {total }}=$ total.

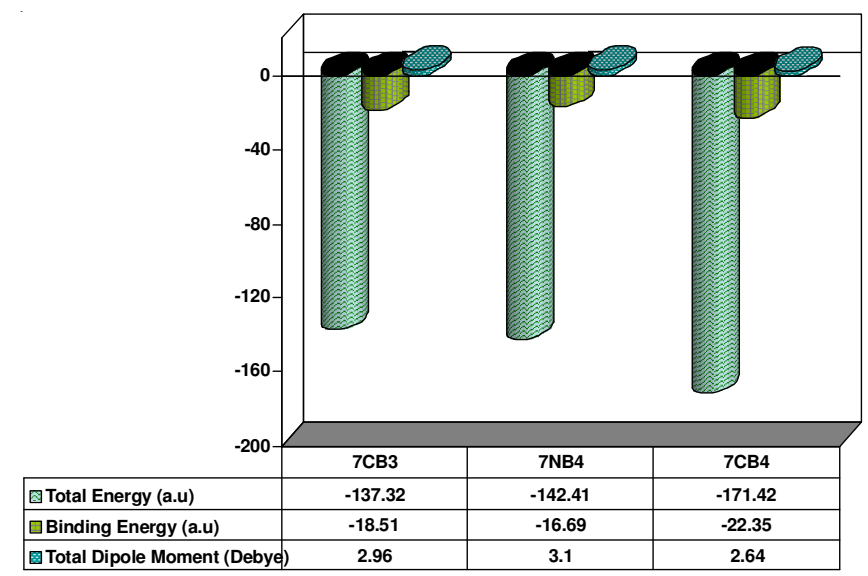

Fig. 2. A comparative picture of molecular parameters of $7 \mathrm{CB} 3,7 \mathrm{NB} 4$ and 7CB4 molecules

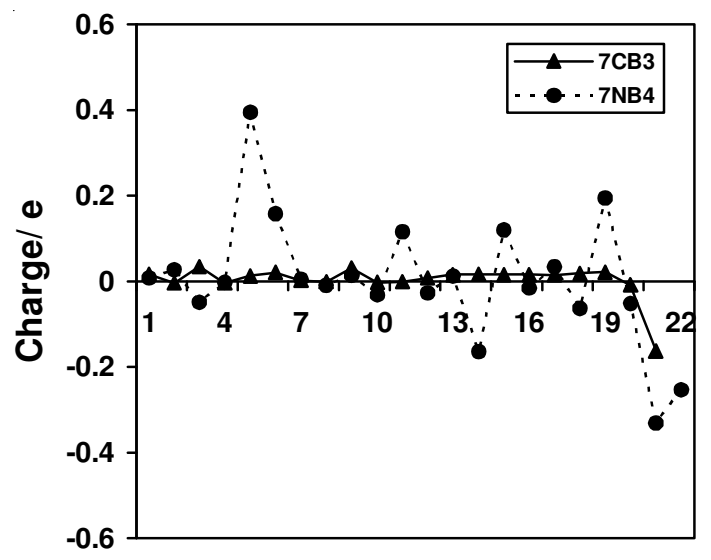

Atom Number

Fig. 3. Partial charges on heavy atoms of 7CB3 and 7NB4

Molecular charge distribution: It is expected that the specific charge distributions in molecules play a decisive role in the formation of various liquid crystal phases. Analysis of molecular charge distribution can deliver good information about local electrostatic interactions which is not possible from experimental point of view. To parameterize the molecular interactions for computational studies atom positioned partial charges are helpful. Quantum chemical computations offer the possibility to take a detailed look at the electronic structure of the molecules. This can be done, by determining atom-based partial charges, which are not quantum mechanical observables. It is noticed from Fig. 3 that even the magnitude of the partial charge is restrained; it varies much from atom to atom. These charges represent the electrostatic molecular interactions very well, but they do not show the real charge distribution in the molecule. Due to the shielding of the carbon charges by the adjacent hydrogens, the correct electrostatic potential might be reproduced by different partial charge distributions. Also, the strong alternation of the charges (Fig. 3) results in a small variation of the electrostatic potential around the molecule. In spite of these uncertainties, the full set of partial charges is very useful, as it can provide a detailed insight into the molecular arrangement in mesophases and they reproduce the electrostatic potential very well. Moreover, high-rank molecular multipoles, polarizabilities and their derivatives can be determined by the interactions of the molecule with the relevant multipole fields. Such molecular multipole properties are associated with the multipole expansion of the electronic charge distribution.

Calculation of interaction energies: The computation of interaction energy during the different modes of interactions is given below:

Stacking interactions: In order to avoid the van der Waals contacts completely, the interacting molecule has been placed at $8 \AA$ along the z-axis with respect to the fixed molecule. The variation of interaction energy components with respect to rotation about $\mathrm{x}$-axis corresponding to configuration y $\left(0^{0}\right) \mathrm{z}$ $\left(0^{0}\right)$ has been carried out. The minimum energy so obtained is then taken as starting point and the entire process is repeated for smaller intervals. The energy has been minimized with respect to translation and rotation about all axes.

An accuracy of $0.1 \AA$ in translation and $1^{0}$ in rotation of one molecule with respect to other has been achieved. The path of minimization strictly depends on the objectives of computation. The study of variation of interaction energy under preselected condition will have completely different path and, therefore, one has to be careful in choosing the specific route. The final lowest stacked geometry after refinement has been obtained with an energy $-13.67 \mathrm{kcal} / \mathrm{mole}$ as shown in Fig. 4. Evidently, the large interaction energy and minimum energy configuration with the long molecular axis almost parallel to one another suggest a strong tendency to maintain the order.

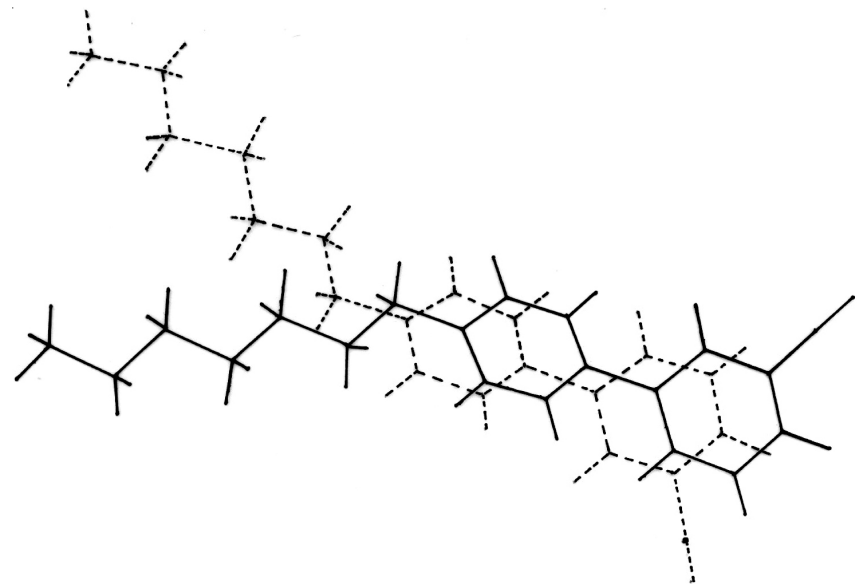

Fig. 4. Lowest energy configuration obtained during stacking interactions with an energy $-13.67 \mathrm{kcal} / \mathrm{mol}$ after refinement 
In-plane interactions: The interacting molecule has been kept at $10 \AA$ separation along the y-axis with respect to the fixed one. The selection of distance has been made to eliminate the possibility of Van der Waals contacts completely and to keep the molecules within short- and medium-range interactions. The variation of various interaction energy components with respect to the translation along $\mathrm{X}$-axis corresponding to configuration y (1800) has been carried out and it has been observed that the dispersion energy component is mainly responsible for the attractions between the pair of $7 \mathrm{CB} 3$ molecule.

Having refined the interacting configuration with respect to translation along $\mathrm{x}$-axis at an equilibrium condition, the energy is brought down and interaction energy components are further investigated with respect to rotation about $\mathrm{x}$-aixs corresponding to configuration y $\left(180^{\circ}\right)$. It has been observed that rotation about $\mathrm{x}$-axis does not alter the configurational energy drastically. The lowest in-plane geometry after refinement has been obtained with energy $-7.79 \mathrm{kcal} / \mathrm{mole}$ (Fig. 5).

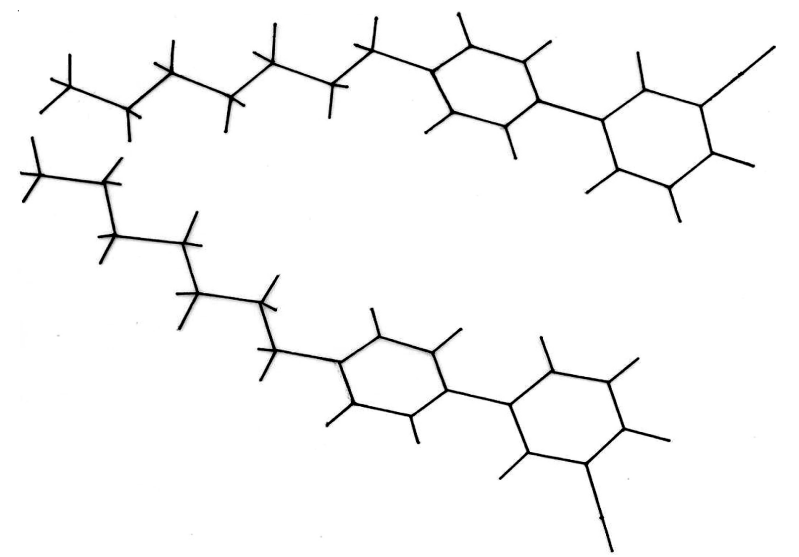

Fig. 5. Lowest energy configuration obtained during in-plane interactions with an energy $-7.79 \mathrm{kcal} / \mathrm{mol}$ after refinement

Terminal interactions: To investigate terminal interactions, the interacting molecule has been shifted by $22 \AA$ with respect to fixed one. It has been observed that the terminal interactions are much weaker than the stacking or in-plane interactions (after due consideration of short contact). The variation of interaction energy components with respect to rotation about $\mathrm{x}$-axis corresponding to configuration y $\left(0^{0}\right)$ has been carried out and it has been observed that rotations about the $\mathrm{x}$-axis show absolutely no preference for any angle i.e., the molecules are free to rotate about their long molecular axis. However, for rotations about $y$-axis, it has been observed that there is slight preference for the molecular axis being on the same line.

Correlation of results: In order to examine the results more closely, the details of stacking and in-plane interaction energy components after refinement are listed in Table- 1 with all contributing terms to enable comparison.

Further, it has been observed (Figs. 4 and 5) that the large interaction energy and minimum energy confirmation with the long molar axis almost parallel to one another suggest a strong tendency to maintain order. Therefore, stacking interactions, which contribute much due to the in-plane and terminal dispersion forces, require much thermal activation and, hence account for the high melting point of the system. At the melting point, thermal agitation will overcome the bindings of the 7CB3 molecules in the crystal and the system will pass directly to an isotropic melt. However, the inherent tendency of the molecules to retain order even after melting does not escape notice. Hence, if suitable functional groups are attached/ replaced to the molecule so that the length to breadth ratio (i.e., 3:1, which seems to be found minimum for mesogens) is altered with dispersion energy, the molecules will show a change in the long-range order, phase transition temperature and other related liquid crystalline properties.

\section{Conclusion}

It may, therefore, be concluded that intermolecular interaction energy calculations are helpful in analyzing the nonmesomorphic behaviour of the molecules in terms of molecular forces accounting for mesomorphism. Further, a comparative study on similar molecules may suggest the factors that cause liquid crystallinity. The present computational approach implies the strong intermolecular interaction energy between a pair of 7CB3 molecules and the specific minimum energy configuration determines the alignment of the molecules with respect to one another. Further, this affords a new way of looking at the substitution of suitable functional groups, which may have an effective role on anisotropic molecular interaction and other factors responsible for liquid crystallinity.

\section{ACKNOWLEDGEMENTS}

The financial support rendered by the DST, CSIR, UGC, New Delhi and DAE-BRNS, Mumbai, India is gratefully acknowledged.

\section{REFERENCES}

1. C. Zannoni, Advances in Computer Simulations of Liquid Crystals, Kluwer Academic, Netherlands (2000).

2. A. Adamski, H. Pauwels and K. Neyts, Liq. Cryst., 31, 997 (2004).

3. S. Özgan, M. Yazici and K. Ates, Asian J. Chem., 23, 3247 (2011).

4. E.R. Soule and A.D. Rey, Liq. Cryst., 38, 201 (2011).

5. S.D. Peroukidis, A.G. Vanakaras and D.J. Photinos, J. Phys. Chem. B, 112, 12761 (2008).

6. P. Lakshmi Praveen and D.P. Ojha, J. Phys. Chem. Solids, 73, 57 (2012).

7. P. Sarkar, S. Paul and P. Mandal, Mol. Cryst. Liq. Cryst., 365, 535 (2001).

8. V.N. Ryzhov, K.I. Guriev and N.N. Nelnichenko, Mol. Cryst. Liq. Cryst., 365, 803 (2001).

9. J.M. Ball and A. Majumdar, Mol. Cryst. Liq. Cryst., 525, 1 (2010).

10. J. Zhang, J. Su and H. Guo, J. Phys. Chem. B, 115, 2214 (2011).

11. C. Bailey and A. Jakli, Phys. Rev. Lett., 99, 207801 (2007).

12. D.P. Ojha and V.G.K.M. Pisipati, Mol. Cryst. Liq. Cryst., 378, 147 (2002).

13. P. Zugenmaier and W. Kuczynski, Mol. Cryst. Liq. Cryst., 457, 93 (2006).

14. J. A. Pople and D.L. Beveridge, Approximate Molecular Orbital Theory, New York: Mc-Graw Hill (1970).

15. P. Claverie, Intermolecular Interactions: From Diatomic to Biopolymers, New York: Wiley (1978).

16. J. Caillet and P. Claverie, Biopolymers, 13, 601 (1974).

17. A.I. Kitaygorodsky and K.V. Mirskay, Kristallografiya, 9, 174 (1964).

18. P.L. Praveen and D.P. Ojha, Mater. Chem. Phys., 126, 248 (2011).

19. P.L. Praveen and D.P. Ojha, J. Mol. Liq., 158, 27 (2011).

20. D.P. Ojha, Z. Naturforsch, 56a, 319 (2001). 